\title{
Measurements of heavy flavours with the ALICE experiment at LHC
}

\author{
Denise Moreira de Godoy* on behalf of the ALICE Collaboration ${ }^{\dagger}$ \\ Institut für Kernphysik \\ Westfälische Wilhelms-Universität Münster \\ Wilhelm-Klemm-Str. 9 - 48149 Münster - Germany \\ E-mail: moreiradeuni-muenster.de
}

\begin{abstract}
The ALICE collaboration has measured the production of open heavy flavours via their hadronic and semi-electronic decays at mid-rapidity and via their semi-muonic decay channel at forward rapidity in pp collisions at $\sqrt{s}=2.76$ and $7 \mathrm{TeV}$, p- $\mathrm{Pb}$ collisions at $\sqrt{s_{N N}}=5.02 \mathrm{TeV}$, and $\mathrm{Pb}-\mathrm{Pb}$ collisions at $\sqrt{s_{N N}}=2.76 \mathrm{TeV}$. We present the ALICE results on the nuclear modification factor, elliptic flow, and azimuthal angular correlations of open heavy-flavour hadrons. The results are compared with model calculations.
\end{abstract}

54th International Winter Meeting on Nuclear Physics 25-29 January 2016

Bormio, Italy

* Speaker.

${ }^{\dagger}$ This work was supported by BMBF (FSP201-ALICE). 


\section{Introduction}

The main goal of the ALICE experiment is to study the state of matter created in ultrarelativistic heavy-ion collisions at the LHC. According to Quantum Chromodynamics (QCD) calculations on the lattice, the energy density and temperature reached in these collisions are sufficiently high for a phase transition of the nuclear matter to a deconfined state of quarks and gluons $[1,2]$. Heavy quarks, i.e. charm and beauty, are mainly produced in hard parton scattering processes at the initial stage of the collisions, because of their large masses. Therefore, they experience the complete evolution of the system created in heavy-ion collisions at high energies, losing energy via radiative and collisional scattering processes in the interaction with the medium constituents. According to QCD calculations, the energy loss of the partons traversing the medium depends on the colour charge and the mass of the parton, leading to a hierarchy where beauty quarks lose less energy than charm quarks, and charm quarks lose less energy than light quarks and gluons $[3,4,5]$.

The in-medium energy loss of heavy quarks can be investigated with the nuclear modification factor $\left(R_{\mathrm{AA}}\right)$ of heavy-flavour hadrons and their decay products, which is defined by the ratio of the yield of particles in heavy-ion collisions with respect to the binary scaled yield in pp collisions. By construction, $R_{\mathrm{AA}}$ is expected to be unity in absence of nuclear effects. The energy loss of heavy flavours in the medium causes a suppression of heavy-flavour particle yields, $R_{\mathrm{AA}}$ $<1$, at intermediate-high transverse momentum $\left(p_{\mathrm{T}}\right)$. The nuclear modification factor is measured in proton-nucleus collisions in order to study the cold nuclear matter (CNM) effects on the heavy-flavour production in heavy-ion collisions as, for instance, nuclear modification of the parton distribution functions $[6,7]$ and possible saturation of the low- $x$ gluon density [8].

The created matter can be studied complementarily with the azimuthal anisotropy of heavyflavour hadrons and their decay products in momentum space. The particle azimuthal distribution in momentum space can be expressed as a Fourier series with respect to the reaction plane (RP), which is defined by the impact parameter direction of the colliding nuclei and the beam direction. In heavy-ion collisions, the second harmonic of the distribution $v_{2}$, called elliptic flow, quantifies the elliptic azimuthal anisotropy of the emitted particles. The elliptic flow of heavy-flavour particles at low $p_{\mathrm{T}}$ is sensitive to the collective motion of heavy quarks in the medium caused by pressure gradients. Because of the almond-shaped overlap area in non-central heavy-ion collisions, particles emitted along the minor axis of the ellipsoid (in-plane direction) traverse less medium on average with respect to particles emitted along the major axis of the ellipsoid (out-of-plane direction). Therefore, the measurement of the $v_{2}$ coefficient of heavy-flavour particles at high $p_{\mathrm{T}}$ can constrain the path-length dependence of the energy loss of heavy quarks.

Further investigation of the medium created in heavy-ion collisions can be provided with the two-particle azimuthal angular correlations involving heavy-flavour particles. The azimuthal angular correlation distributions in heavy-ion collisions is sensitive to the nuclear modification of heavy quark fragmentation functions. The measurement in proton-nucleus collisions can be used to investigate CNM effects and the potential existence of collective phenomena in such collisions [9]. In addition, the azimuthal angular correlations between electrons from heavy-flavour decays and charged particles in pp collisions can be used to estimate the relative contribution of electrons from beauty-hadron decays to the yield of electrons from heavy-flavour decays. 


\section{ALICE experiment and particle identification}

The ALICE detector consists of various detectors for particle reconstruction, triggering and event characterization. In the following, only the ALICE subsystems that are relevant for the presented open heavy-flavour analyses are described. More details of the ALICE detector can be found in $[10,11]$.

Charged particles are reconstructed and identified with the Inner Tracking System (ITS), the Time Projection Chamber (TPC), the Time-Of-Flight (TOF) detectors at mid-rapidity $(|\eta|<0.9)$. Electrons are also identified with the ElectroMagnetic Calorimeter (EMCal) at mid-rapidity $(|\eta|<$ 0.7). Muons are reconstructed and identified with the Muon Spectrometer at forward rapidity $(-4<\eta<-2.5)$. The V0 detector, which consists of the scintillator counters V0A at backward rapidity $(2.8<\eta<5.1)$ and V0C at forward rapidity $(-3.7<\eta<-1.7)$, and the Zero Degree Calorimeters (ZDC) are used for triggering and event selection. The V0 detector is also used to estimate the reaction plane direction of the event.

The production of open heavy flavours has been measured with ALICE via their hadronic and semi-electronic decays at mid-rapidity and via their semi-muonic decay channel at forward rapidity in pp collisions at $\sqrt{s}=2.76$ and $7 \mathrm{TeV}, \mathrm{p}-\mathrm{Pb}$ collisions at $\sqrt{s_{N N}}=5.02 \mathrm{TeV}$, and $\mathrm{Pb}-\mathrm{Pb}$ collisions at $\sqrt{s_{N N}}=2.76 \mathrm{TeV}$.

Charm mesons are reconstructed via the following hadronic decay channels: $\mathrm{D}^{0} \rightarrow \mathrm{K}^{-} \pi^{+}$, $\mathrm{D}^{+} \rightarrow \mathrm{K}^{-} \pi^{+} \pi^{+}, \mathrm{D}^{\star+} \rightarrow D^{0}\left(\rightarrow \mathrm{K}^{-} \pi^{+}\right) \pi^{+}, \mathrm{D}_{s}^{+} \rightarrow \phi\left(\rightarrow \mathrm{K}^{-} \mathrm{K}^{+}\right) \pi^{+}$, and their charge conjugates. D-meson decay particles are reconstructed and identified with the ITS, TPC and TOF detectors. The decay topologies are reconstructed based on the displacement of the decay particles with respect to the primary vertex, for instance, the separation between the primary and secondary vertices and the pointing angle of the reconstructed D-meson momentum [12]. The D-meson yields are extracted from fits to the invariant mass distributions for $\mathrm{D}^{0}, \mathrm{D}^{+}$and $\mathrm{D}_{s}^{+}$candidates and from mass difference $\Delta M=M(\mathrm{~K} \pi \pi)-M(\mathrm{~K} \pi)$ distributions for $\mathrm{D}^{\star+}$ candidates. The contribution of the secondary D mesons from B-meson decays is estimated using perturbative QCD (pQCD) calculations of the B-meson production [13] and Monte Carlo (MC) simulations.

The electron candidates are reconstructed with the ITS, TPC, TOF and EMCal detectors. The electron sample is composed of electrons from heavy-flavour hadron decays and from background sources, which are mainly electrons from photon conversions in detector material and from Dalitz decays of neutral mesons. The contribution of electrons that do not originate from heavy-flavour hadron decays is obtained using the invariant mass of electron-positron pairs, which is expected to have small values for pairs coming from photon conversions and Dalitz decays, and the cocktail method, which uses simulations of the background electron contributions based on the measurement of cross sections of the background electron sources [14]. The selection of electrons from beauty-hadron decays is based on the track impact parameter $\left(d_{0}\right)$, defined as the distance of closest approach between the charged track and the interaction vertex in the transverse plane, that allows to statistically separate electrons from different sources. The contribution of electrons from beautyhadron decays in $\mathrm{Pb}-\mathrm{Pb}$ collisions is extracted from fits to the $d_{0}$ distributions of the measured electrons based on MC simulations of the distributions of each electron source. In pp collisions, the contribution of electrons from beauty-hadron decays is obtained with a requirement on the minimum $d_{0}$ and the contribution of the remaining electrons that do not originate from beauty-hadron 
decays is obtained with the cocktail method.

The muon candidates are reconstructed with the muon spectrometer. The muon sample includes muons from heavy-flavour hadron decays and muons from background sources, which are mainly $\pi$ and K decays. Background muons are estimated in pp collisions via MC simulations, while the estimation in $\mathrm{p}-\mathrm{Pb}$ and $\mathrm{Pb}-\mathrm{Pb}$ collisions is obtained using simulations of the decays of $\pi$ and $\mathrm{K}$, with the $p_{\mathrm{T}}$ distributions of these hadrons tuned to match the measurements at mid-rapidity and then extrapolated to the rapidity interval $2.5<y<4$ [15]. The forward-backward asymmetry of charged particles measured by the CMS Collaboration [16] is used to constrain the rapidity extrapolation in the analysis in $\mathrm{p}-\mathrm{Pb}$ collisions. The contribution of muons from $\mathrm{W}$ decays, which is not negligible at high $p_{\mathrm{T}}$, is subtracted statistically in the $R_{\mathrm{AA}}$ analysis using MC simulations.

\section{Results}

For all results presented here, the vertical error bars represent the statistical uncertainties, the horizontal error bars indicate the bin widths and the empty boxes represent the total systematic uncertainties. The boxes centered at unity in the nuclear modification factor figures represent the normalization uncertainty.

\subsection{Nuclear modification factor}

The nuclear modification factors in $\mathrm{Pb}-\mathrm{Pb}$ collisions $\left(R_{\mathrm{AA}}\right)$ and in $\mathrm{p}-\mathrm{Pb}$ collisions $\left(R_{\mathrm{pPb}}\right)$ are defined as:

$$
R_{\mathrm{AA}}\left(p_{\mathrm{T}}\right)=\frac{1}{\left\langle T_{\mathrm{AA}}\right\rangle} \frac{\mathrm{d} N_{\mathrm{AA}} / \mathrm{d} p_{\mathrm{T}}}{\mathrm{d} \sigma_{\mathrm{pp}} / \mathrm{d} p_{\mathrm{T}}}, R_{\mathrm{pPb}}\left(p_{\mathrm{T}}\right)=\frac{1}{A} \frac{\mathrm{d} \sigma_{\mathrm{pPb}} / \mathrm{d} p_{\mathrm{T}}}{\mathrm{d} \sigma_{\mathrm{pp}} / \mathrm{d} p_{\mathrm{T}}},
$$

where $\mathrm{d} N_{\mathrm{AA}} / \mathrm{d} p_{\mathrm{T}}$ is the $p_{\mathrm{T}}$-differential yield in $\mathrm{Pb}-\mathrm{Pb}$ collisions, $T_{\mathrm{AA}}$ is the nuclear overlap function in $\mathrm{Pb}-\mathrm{Pb}$ collisions, which is evaluated with the Glauber model [17], $A$ is the mass number of the $\mathrm{Pb}$ nucleus, $\mathrm{d} \sigma_{\mathrm{pp}} / \mathrm{d} p_{\mathrm{T}}$ and $\mathrm{d} \sigma_{\mathrm{pPb}} / \mathrm{d} p_{\mathrm{T}}$ are the $p_{\mathrm{T}}$-differential cross sections in pp and $\mathrm{p}-\mathrm{Pb}$ collisions, respectively. Depending on the analysis, the pp references for the $R_{\mathrm{AA}}$ and $R_{\mathrm{pPb}}$ measurements are obtained by the measured cross sections in pp collisions at the same centre-of-mass energy or by a $\sqrt{s}$-scaling [18] of the measured cross sections in pp collisions at $\sqrt{s}=2.76$ and/or $7 \mathrm{TeV}$. More details concerning the analyses can be found in [12, 19, 20].

Figure 1 shows the nuclear modification factor of prompt $\mathrm{D}$ mesons (average of $\mathrm{D}^{0}, \mathrm{D}^{+}$and $\left.\mathrm{D}^{*+}\right)$ as a function of $p_{\mathrm{T}}$ in minimum-bias $\mathrm{p}-\mathrm{Pb}$ collisions at $\sqrt{s_{\mathrm{NN}}}=5.02 \mathrm{TeV}$ and in $\mathrm{Pb}-\mathrm{Pb}$ collisions at $\sqrt{s_{\mathrm{NN}}}=2.76 \mathrm{TeV}$ in the $0-10 \%$ and $30-50 \%$ centrality classes [12]. No significant modification of the $\mathrm{D}$-meson spectrum is observed in $\mathrm{p}-\mathrm{Pb}$ collisions relative to $\mathrm{pp}$ collisions for $p_{\mathrm{T}}>2 \mathrm{GeV} / c$, while a strong suppression of $\mathrm{D}$ mesons is observed in central and semi-central $\mathrm{Pb}-\mathrm{Pb}$ collisions for $p_{\mathrm{T}}>3 \mathrm{GeV} / c$. Therefore, the suppression observed in $\mathrm{Pb}-\mathrm{Pb}$ collisions is predominantly due to final-state effects of the strongly-interacting matter created in those collisions.

Figure 2 shows the $R_{\mathrm{AA}}$ of $\mathrm{D}$ mesons (average of $\mathrm{D}^{0}, \mathrm{D}^{+}$and $\mathrm{D}^{*+}$ ) at mid-rapidity $(|y|<$ $0.5)$ and in the interval $8<p_{\mathrm{T}}<16 \mathrm{GeV} / c$ as a function of the average number of nucleons participating in the collision in each multiplicity class $\left\langle N_{\text {part }}\right\rangle$ [12], which represents the collision centrality. The result is compared with the $R_{\mathrm{AA}}$ of charged pions (left) at mid-rapidity $(|y|<0.8)$ and in the same $p_{\mathrm{T}}$ interval $\left(8<p_{\mathrm{T}}<16 \mathrm{GeV} / c\right)$ [21] and with the $R_{\mathrm{AA}}$ of non-prompt $\mathrm{J} / \psi$ from B-meson decays (right) measured by the CMS Collaboration [22] in wider intervals of rapidity 


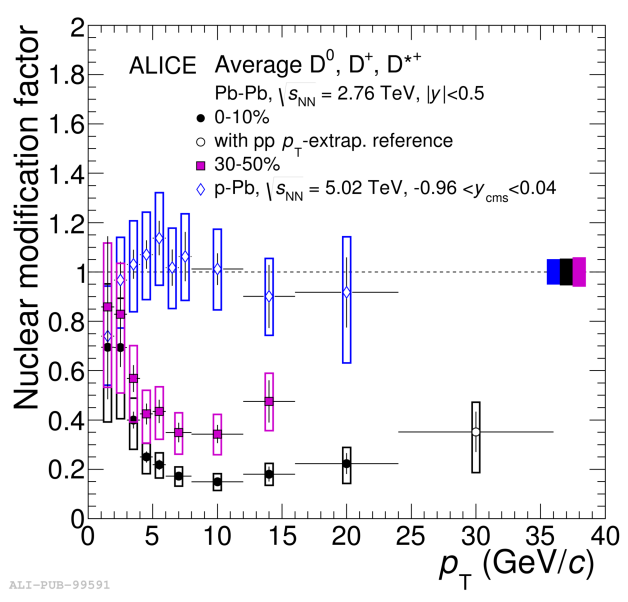

Figure 1: Nuclear modification factor of prompt $\mathrm{D}$ mesons (average of $\mathrm{D}^{0}, \mathrm{D}^{+}$and $\mathrm{D}^{*+}$ ) as a function of $p_{\mathrm{T}}$ in $\mathrm{p}-\mathrm{Pb}$ collisions at $\sqrt{s_{\mathrm{NN}}}=5.02 \mathrm{TeV}$ and in $\mathrm{Pb}-\mathrm{Pb}$ collisions at $\sqrt{s_{\mathrm{NN}}}=2.76 \mathrm{TeV}$ in the $0-10 \%$ and $30-50 \%$ centrality classes [12].

$(|y|<1.2)$ and transverse momentum $\left(6.5<p_{\mathrm{T}}<30 \mathrm{GeV} / c\right)$. The centrality dependence of the D-meson $R_{\mathrm{AA}}$ shows an increase of the suppression of $\mathrm{D}$ mesons towards central collisions (large $\left\langle N_{\text {part }}\right\rangle$ values), which is consistent with the qualitative expectation of the parton in-medium energy loss. The observed D-meson suppression is compatible, within uncertainties, with the observed suppression of charged pions in the same $p_{\mathrm{T}}$ interval, while it is found to be larger compared with the observed non-prompt $\mathrm{J} / \psi$ suppression. The comparison of $R_{\mathrm{AA}}$ of $\mathrm{D}$ mesons and nonprompt $\mathrm{J} / \psi$ from B-hadron decays hints at a difference in the suppression of particles originating from charm and beauty quarks in the most central collisions. The results are described by model calculations $[23,24]$ that include colour charge and mass dependence of the in-medium parton energy loss via radiative and collisional processes. The non-prompt $\mathrm{J} / \psi R_{\mathrm{AA}}$ obtained using the charm-quark mass in the calculation of the energy loss of beauty quarks is used as a test for the origin of the difference in the $R_{\mathrm{AA}}$ of D mesons and non-prompt $\mathrm{J} / \psi$. The corresponding result is closer to the D-meson $R_{\mathrm{AA}}$, indicating that the observed difference in this model is predominantly due to the quark mass dependence of the parton energy loss.

Figure 3 shows the $p_{\mathrm{T}}$ dependence of the nuclear modification factor of electrons from heavyflavour hadron decays in the interval $-1.06<y_{\mathrm{cms}}<0.14$ [19] (left) and of muons from heavyflavour hadron decays in the intervals $2.5<y_{\mathrm{cms}}<3.54$ and $-4<y_{\mathrm{cms}}<-2.96$ (right) in $\mathrm{p}-\mathrm{Pb}$ collisions at $\sqrt{s_{\mathrm{NN}}}=5.02 \mathrm{TeV}$. The convention used for the rapidity sign in $\mathrm{p}-\mathrm{Pb}$ collisions is negative for $\mathrm{Pb}$-going direction, which addresses the orientation of the muon spectrometer, and positive for p-going one. No significant modification of the spectra of leptons from heavy-flavour hadron decays is observed in $\mathrm{p}-\mathrm{Pb}$ collisions relative to $\mathrm{pp}$ collisions. The model calculations including only initial-state effects $[13,25,26,27,28]$ describe the $p_{\mathrm{T}}$ dependence of the $R_{\mathrm{pPb}}$ of electrons from heavy-flavour hadron decays.

Figure 4 (left panel) shows the $p_{\mathrm{T}}$ dependence of the $R_{\mathrm{AA}}$ of electrons from heavy-flavour hadron decays at mid-rapidity $(|y|<0.6)$ and muons from heavy-flavour hadron decays at forward rapidity $(2.5<y<4)$ in $\mathrm{Pb}-\mathrm{Pb}$ collisions at $\sqrt{s_{\mathrm{NN}}}=2.76 \mathrm{TeV}$ in the $0-10 \%$ centrality class [20]. A strong suppression of electrons and muons from heavy-flavour hadron decays is observed for 

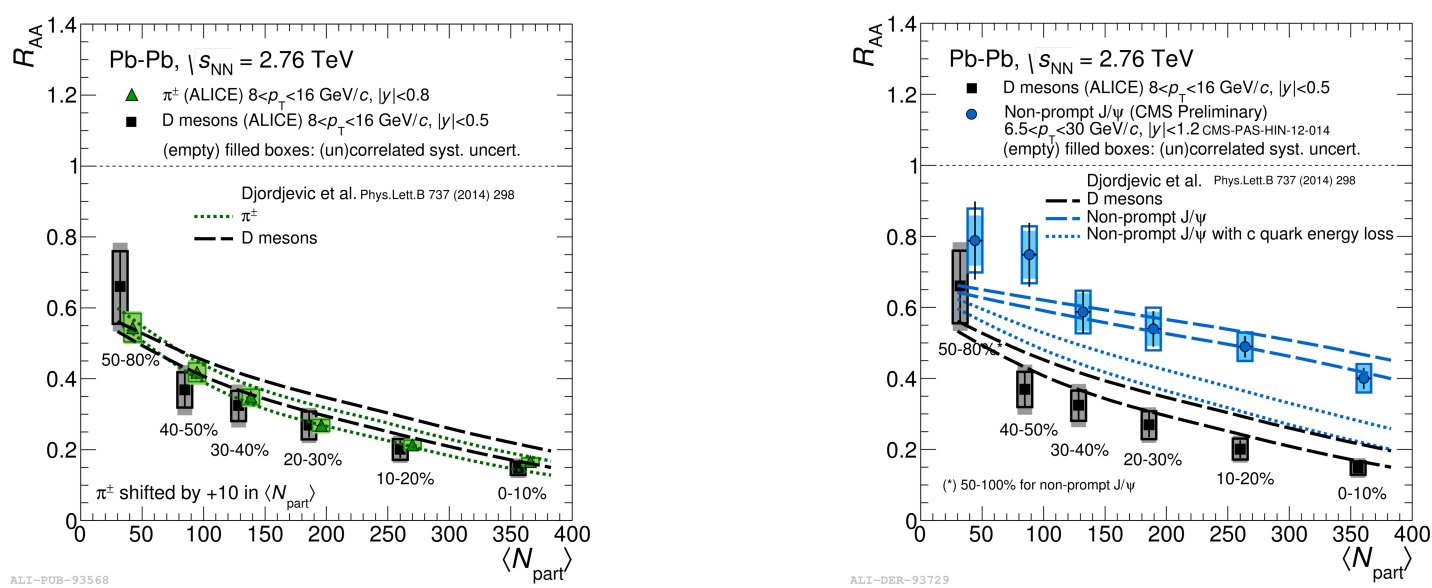

Figure 2: D-meson $R_{\mathrm{AA}}$ at mid-rapidity $(|y|<0.5)$ in the interval $8<p_{\mathrm{T}}<16 \mathrm{GeV} / c$ as a function of $\left\langle N_{\text {part }}\right\rangle$ and centrality class [12] compared with the $\pi^{ \pm} R_{\mathrm{AA}}$ at mid-rapidity $(|y|<0.8)$ in the interval $8<p_{\mathrm{T}}<16$ $\mathrm{GeV} / c$ [21] (left) and with the non-prompt $\mathrm{J} / \psi R_{\mathrm{AA}}$ measured by the CMS Collaboration in the intervals $|y|<1.2$ and $6.5<p_{\mathrm{T}}<30 \mathrm{GeV} / c$ [22] (right). The results are compared with model calculations [23, 24].
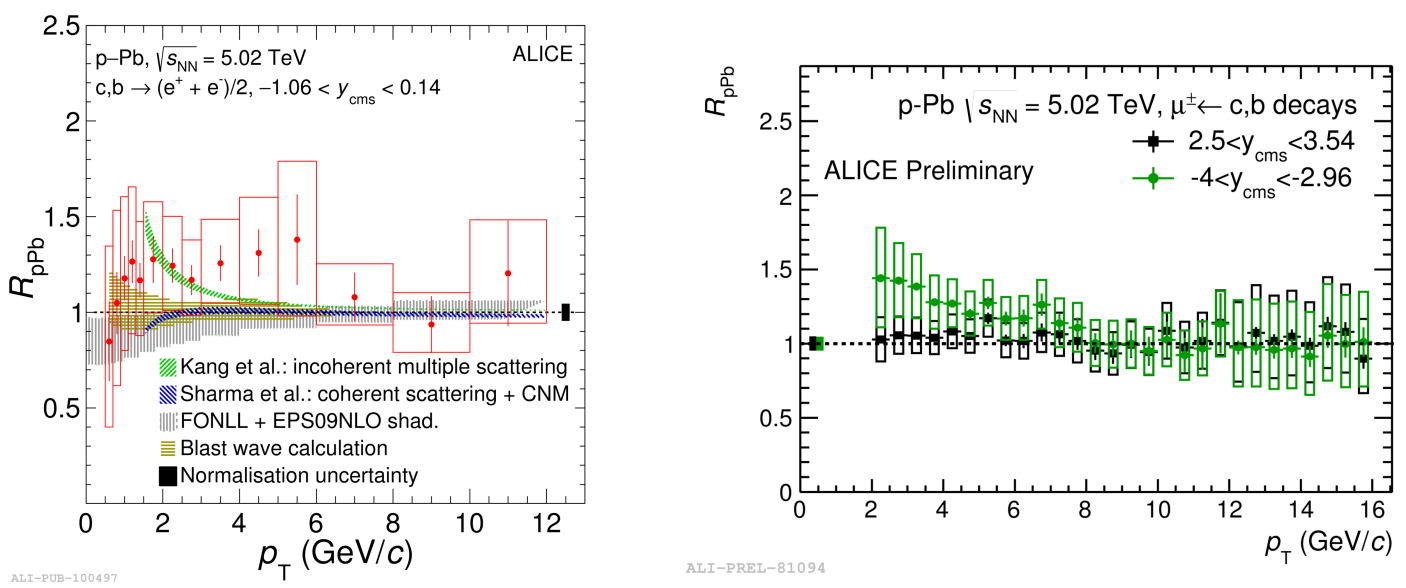

Figure 3: Left panel: $R_{\mathrm{pPb}}$ of electrons from heavy-flavour hadron decays as a function of $p_{\mathrm{T}}$ in the interval $-1.06<y_{\mathrm{cms}}<0.14$ in minimum-bias $\mathrm{p}-\mathrm{Pb}$ collisions at $\sqrt{s_{\mathrm{NN}}}=5.02 \mathrm{TeV}$ [19] compared with theoretical models $[13,25,26,27,28]$. Right panel: $R_{\mathrm{pPb}}$ of muons from heavy-flavour hadron decays as a function of $p_{\mathrm{T}}$ in the intervals $2.5<y_{\mathrm{cms}}<3.54$ and $-4<y_{\mathrm{cms}}<-2.96$ in $\mathrm{p}-\mathrm{Pb}$ collisions at $\sqrt{s_{\mathrm{NN}}}=5.02 \mathrm{TeV}$.

$p_{\mathrm{T}}>3 \mathrm{GeV} / c$. The observed suppression in central $\mathrm{Pb}-\mathrm{Pb}$ collisions is predominantly due to the final-state effects of the created medium since the suppression cannot be explained by CNM effects, as shown in Figure 3. The $p_{\mathrm{T}}$ dependence of the $R_{\mathrm{AA}}$ of electrons from beauty-hadron decays at mid-rapidity $(|y|<0.8)$ in $\mathrm{Pb}-\mathrm{Pb}$ collisions at $\sqrt{s_{\mathrm{NN}}}=2.76 \mathrm{TeV}$ in the $0-20 \%$ centrality class is shown in the right panel of Figure 4. The observed suppression of electrons from beauty-hadron decays for $p_{\mathrm{T}}>3 \mathrm{GeV} / c$ provides a hint for beauty quark in-medium energy loss.

\subsection{Elliptic flow}

The measurements of the elliptic flow of D mesons and electrons from heavy-flavour hadron decays presented here are performed with the event plane method, where the reaction plane angle 

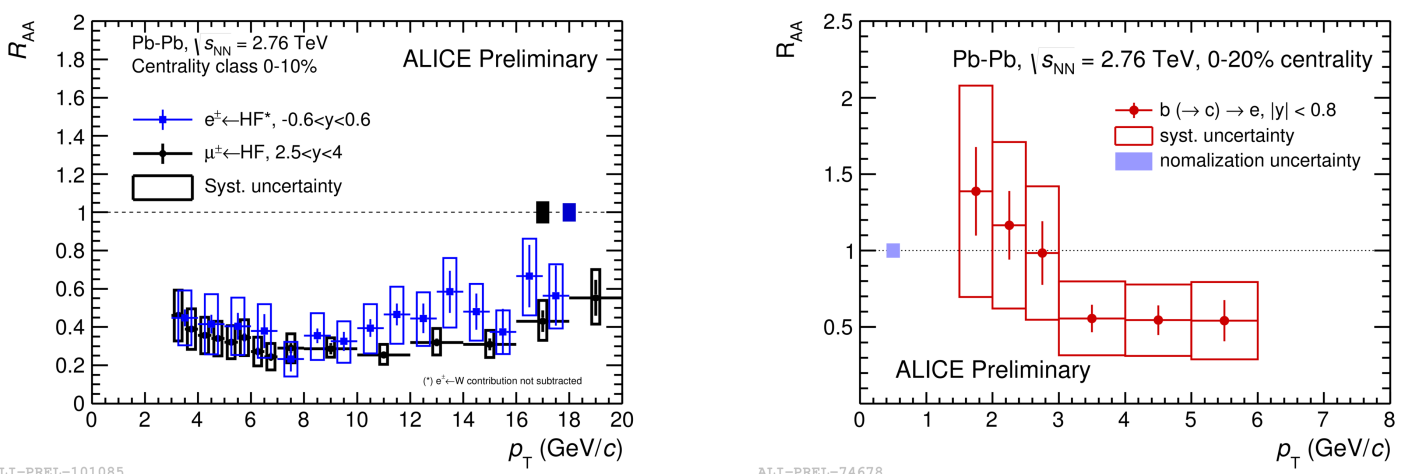

Figure 4: Left panel: $R_{\mathrm{AA}}$ of electrons from heavy-flavour hadron decays at mid-rapidity $(|y|<0.6)$ and muons from heavy-flavour hadron decays at forward rapidity $(2.5<y<4)$ as a function of $p_{\mathrm{T}}$ in $\mathrm{Pb}-\mathrm{Pb}$ collisions at $\sqrt{s_{\mathrm{NN}}}=2.76 \mathrm{TeV}$ in the $0-10 \%$ centrality class. Right panel: $R_{\mathrm{AA}}$ of electrons from beautyhadron decays at mid-rapidity $(|y|<0.8)$ as a function of $p_{\mathrm{T}}$ in $\mathrm{Pb}-\mathrm{Pb}$ collisions at $\sqrt{s_{\mathrm{NN}}}=2.76 \mathrm{TeV}$ in the $0-20 \%$ centrality class.

is estimated from the symmetry angle of the particle azimuthal distribution in the transverse plane. Depending on the analysis, the $v_{2}$ coefficient is obtained by:

$$
v_{2}\{\mathrm{EP}\}=\frac{1}{\mathrm{R}_{2}}\left\langle\cos \left[2\left(\varphi-\Psi_{2}\right)\right]\right\rangle \text { or } \mathrm{v}_{2}\{\mathrm{EP}\}=\frac{1}{\mathrm{R}_{2}} \frac{\pi}{4} \frac{\mathrm{N}_{\text {in }}-\mathrm{N}_{\text {out }}}{\mathrm{N}_{\text {in }}+\mathrm{N}_{\text {out }}}
$$

where $\varphi$ is the azimuthal angle of the heavy-flavour particles, $\Psi_{2}$ is the symmetry plane angle, $R_{2}$ is the event plane resolution correction, the angle brackets represent the average over the heavyflavour particles in all events, and the factors $N_{\text {in }}$ and $N_{\text {out }}$ are the number of heavy-flavour particles along the in- and out-plane directions, respectively. The presented measurement of the elliptic flow of muons from heavy-flavour hadron decays is performed with the two-particle Q cumulants. In the case of the leptons from heavy-flavour hadron decays, the $v_{2}$ of the background leptons is estimated and the contribution is subtracted from the measured $v_{2}$ of inclusive leptons. More analysis details can be found in $[15,29,30,31]$.

Figure 5 shows the $v_{2}$ of prompt $\mathrm{D}^{0}$ mesons at mid-rapidity $(|y|<0.8)$ as a function of $p_{\mathrm{T}}$ in three centrality classes $\left(0-10 \%, 10-30 \%\right.$, and 30-50\%) in $\mathrm{Pb}-\mathrm{Pb}$ collisions at $\sqrt{s_{\mathrm{NN}}}=2.76$ $\mathrm{TeV}[29,30]$. A positive $v_{2}$ is observed with more than $5 \sigma$ significance in the interval $2<p_{\mathrm{T}}<$ $6 \mathrm{GeV} / c$ in the $30-50 \%$ centrality class, which indicates a collective motion of low- $p_{\mathrm{T}}$ charm quarks in the medium. The centrality dependence of $v_{2}$ of prompt $\mathrm{D}^{0}$ mesons hints for an increase of $v_{2}$ from central to peripheral collisions, which is consistent with the qualitative expectation of the evolution of the geometrical anisotropy of the overlap region of the colliding nuclei with the centrality. The prompt $\mathrm{D}^{0}$-meson $v_{2}$ is compatible, within the uncertainties, with the chargedparticle $v_{2}$ in the same centrality classes.

Figure 6 shows the $v_{2}$ of electrons from heavy-flavour hadron decays obtained with the event plane method at mid-rapidity $(|y|<0.7)$ and muons from heavy-flavour hadron decays obtained with the two-particle $\mathrm{Q}$ cumulant method at forward rapidity $(2.5<y<4)[15]$ as a function of $p_{\mathrm{T}}$ in three centrality classes $\left(0-10 \%, 10-20 \%\right.$, and 20-40\%) in Pb-Pb collisions at $\sqrt{s_{\mathrm{NN}}}=2.76 \mathrm{TeV}$. A positive $v_{2}$ is observed with more than $3 \sigma$ significance in the interval $1<p_{\mathrm{T}}^{\mathrm{e} \leftarrow H F}<3 \mathrm{GeV} / c$ for 


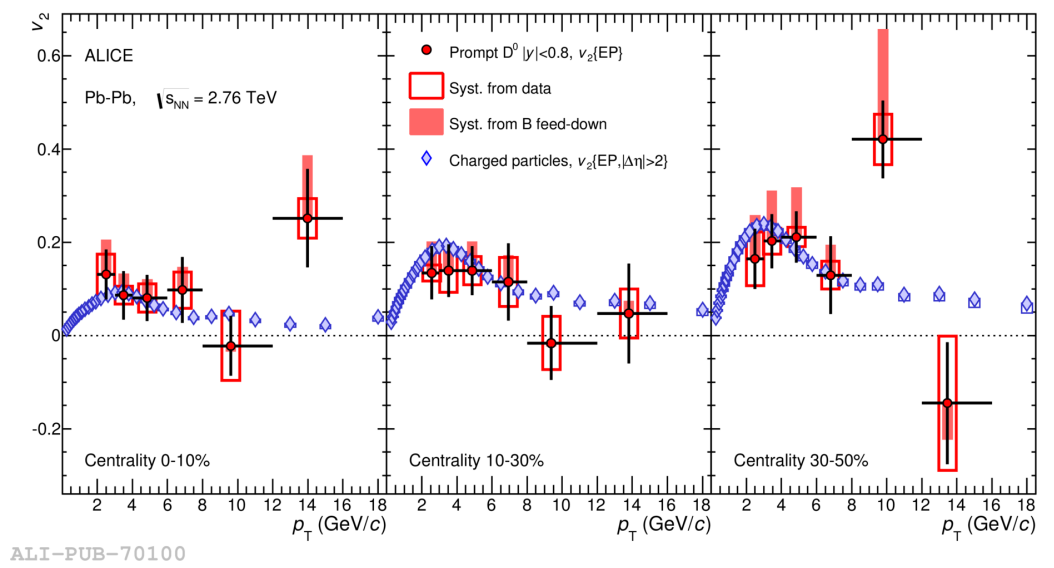

Figure 5: $\quad v_{2}$ of prompt $\mathrm{D}^{0}$ mesons at mid-rapidity $(|y|<0.8)$ as a function of $p_{\mathrm{T}}$ in $\mathrm{Pb}-\mathrm{Pb}$ collisions at $\sqrt{s_{\mathrm{NN}}}=2.76 \mathrm{TeV}$ in the $0-10 \%$ (left), 10-30\% (central), and 30-50\% (right) centrality classes [29, 30]. Results are compared with the charged-particle $v_{2}$ for the same centrality classes.

electrons from heavy-flavour decays and in the interval $3<p_{\mathrm{T}}^{\mu \leftarrow H F}<5.5 \mathrm{GeV} / c$ for muons from heavy-flavour decays in the $20-40 \%$ centrality class, indicating that heavy quarks participate in the collective motion of the medium. Results also suggest an increasing of $v_{2}$ towards more peripheral collisions.

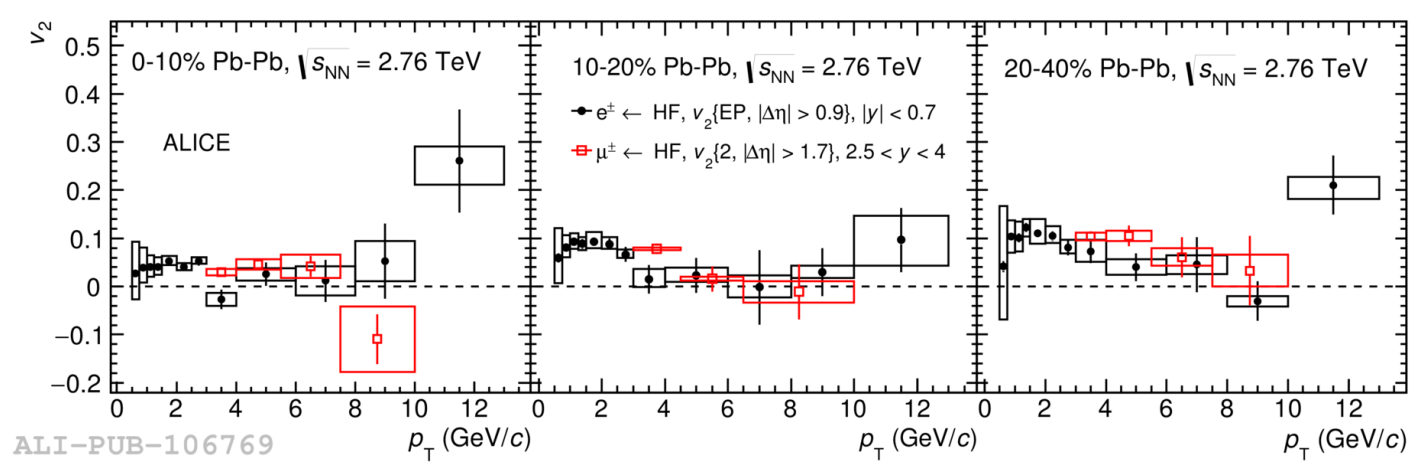

Figure 6: $v_{2}$ of electrons from heavy-flavour hadron decays at mid-rapidity $(|y|<0.7)[31]$ and muons from heavy-flavour hadron decays at forward rapidity $(2.5<y<4)[15]$ as a function of $p_{\mathrm{T}}$ in $\mathrm{Pb}-\mathrm{Pb}$ collisions at $\sqrt{s_{\mathrm{NN}}}=2.76 \mathrm{TeV}$ in the $0-10 \%$ (left), 10-20\% (central) and 20-40\% (right) centrality classes.

Figure 7 shows the comparison of $v_{2}$ (left) and $R_{\mathrm{AA}}$ (right) of electrons from heavy-flavour hadron decays as a function of $p_{\mathrm{T}}$ measured in $\mathrm{Pb}-\mathrm{Pb}$ collisions in the $20-40 \%$ and $0-10 \%$ centrality classes, respectively, with model calculations [32, 33, 34, 35]. The models including interactions of the heavy quarks with the medium constituents can describe qualitatively the $v_{2}$ of electrons from heavy-flavour hadron decays. The simultaneous comparison of $R_{\mathrm{AA}}$ and $v_{2}$ measurements to model calculations provides constraints for the models.

\subsection{Azimuthal angular correlations}

The azimuthal angular correlation between heavy-flavour particles (trigger) and charged par- 

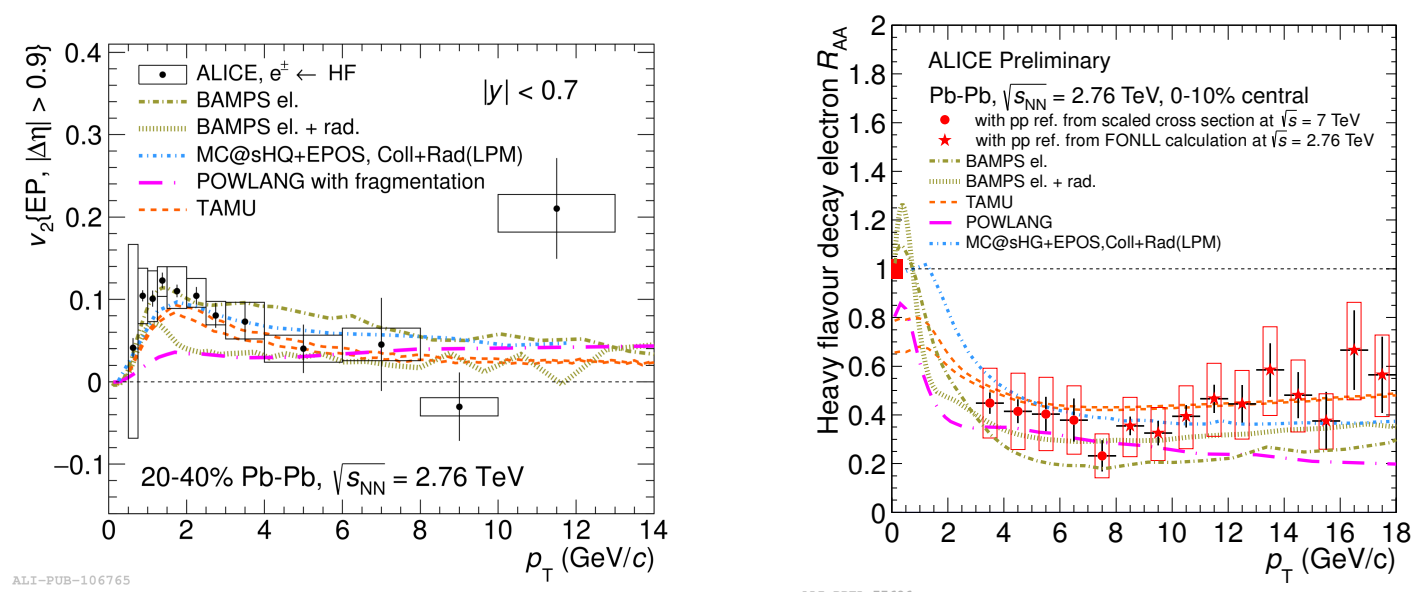

Figure 7: Left panel: $v_{2}$ of electrons from heavy-flavour hadron decays at mid rapidity $(|y|<0.7)$ as a function of $p_{\mathrm{T}}$ measured in $\mathrm{Pb}-\mathrm{Pb}$ collisions at $\sqrt{s_{\mathrm{NN}}}=2.76 \mathrm{TeV}$ in the 20-40\% centrality class [31]. Right panel: $R_{\mathrm{AA}}$ of electrons from heavy-flavour hadron decays at mid rapidity $(|y|<0.6)$ as a function of $p_{\mathrm{T}}$ measured in $\mathrm{Pb}-\mathrm{Pb}$ collisions at $\sqrt{s_{\mathrm{NN}}}=2.76 \mathrm{TeV}$ in the $0-10 \%$ centrality class. For both panels, results are compared with parton transport models [32, 33, 34, 35].

ticles (associated) is defined as the differential yield of trigger-associated particle pairs in azimuth $(\Delta \varphi)$ and in pseudorapidity $(\Delta \eta)$ :

$$
C(\Delta \varphi, \Delta \eta)=\frac{1}{N_{\text {trigger }}} \frac{\mathrm{d}^{2} N_{\text {pair }}}{\mathrm{d} \Delta \varphi \mathrm{d} \Delta \eta}
$$

where $N_{\text {trigger }}$ is the number of trigger particles and $N_{\text {pair }}$ is the number of trigger-associated particle pairs. The correlation distributions are corrected for the limited acceptance and spatial inhomogeneities of the detector using the event mixing technique and for the efficiency of the trigger and associated particle reconstructions. Details of the analyses can be found in [36, 37].

Figure 8 (left panel) shows the azimuthal angular correlation between electrons from heavyflavour hadron decays in two intervals of pseudorapidity $(|\eta|<0.9$ and $|\eta|<0.7)$ and transverse momentum $\left(1.5<p_{\mathrm{T}}<2.5 \mathrm{GeV} / c\right.$ and $\left.4.5<p_{\mathrm{T}}<6 \mathrm{GeV} / c\right)$ and charged hadrons for $p_{\mathrm{T}}>0.3$ $\mathrm{GeV} / c$ in pp collisions at $\sqrt{s}=2.76 \mathrm{TeV}$ [36]. The near $(\Delta \varphi \approx 0)$ and away side $(\Delta \varphi \approx \pi)$ peaks are clearly visible. The relative contribution of electrons from beauty-hadron decays to the yield of electrons from heavy-flavour decays $\left(r_{b}\right)$ is obtained with a fit to the measured correlation distributions using a MC simulation with PYTHIA [38] of the correlation distributions of electrons from beauty and charm hadron decays, as shown in the left panel of Figure 8 . The width of the near-side peak is larger for electrons from beauty-hadron decays than from charm-hadron decays due to the different mass and decay kinematics of charm and beauty hadrons. The factor $r_{b}$ is shown in the top right panel of Figure 8 and compared with the result obtained using the impact parameter method and predictions from different pQCD calculations $[13,39,40]$. The contribution of electrons from beauty-hadron decays to the yield of electrons from heavy-flavour decays increases with $p_{\mathrm{T}}$ and reaches $50 \%$ at approximately $4 \mathrm{GeV} / c$. The $r_{b}$ parameter is used to scale the measured heavyflavour decay electron cross section in pp collisions at $\sqrt{s}=2.76 \mathrm{TeV}$ [41] in order to obtain the cross section of electrons from beauty hadron decays. The corresponding result (bottom right panel 
of Figure 8) shows a good agreement with the one obtained using the impact parameter method.
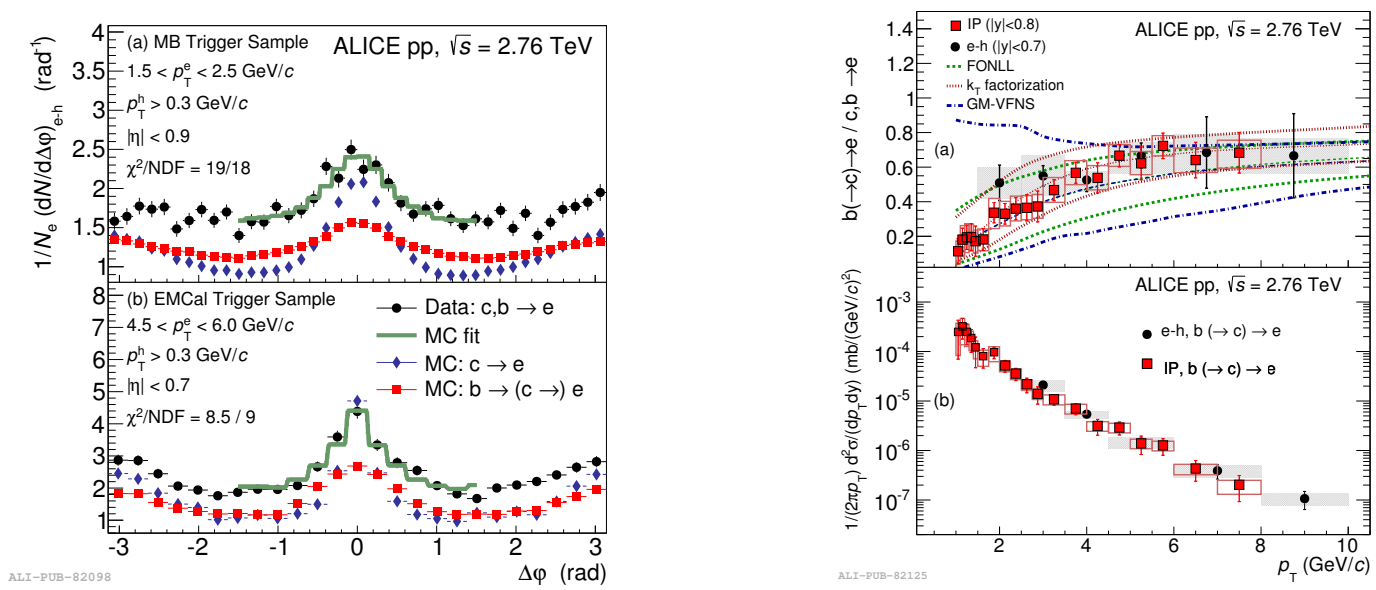

Figure 8: Left panel: azimuthal angular correlation between electrons from heavy-flavour hadron decays in two intervals of pseudorapidity $(|\eta|<0.9$ and $|\eta|<0.7)$ and transverse momentum $\left(1.5<p_{\mathrm{T}}<2.5\right.$ $\mathrm{GeV} / c$ and $4.5<p_{\mathrm{T}}<6 \mathrm{GeV} / c$ ) and charged hadrons for $p_{\mathrm{T}}>0.3 \mathrm{GeV} / c$ in pp collisions at $\sqrt{s}=2.76$ TeV. Right panel: (a) relative contribution of electrons from beauty-hadron decays to the heavy-flavour decay electron yield $\left(r_{b}\right)$ compared with the result obtained using the impact parameter method and different pQCD calculations $[13,39,40]$. (b) $p_{\mathrm{T}}$-differential inclusive production cross section of electrons from beauty-hadron decays compared with the result obtained using the impact parameter method.

Figure 9 shows the azimuthal correlation of $\mathrm{D}$ mesons in the $p_{\mathrm{T}}$ interval $5<p_{\mathrm{T}}<8 \mathrm{GeV} / c$ and charged particles with $p_{\mathrm{T}}>0.3 \mathrm{GeV} / c$ in $\mathrm{p}-\mathrm{Pb}$ collisions at $\sqrt{s_{\mathrm{NN}}}=5.02 \mathrm{TeV}$ and in pp collisions at $\sqrt{s}=7 \mathrm{TeV}$. The correlation pattern in pp collisions is compatible with the one measured in $\mathrm{p}-\mathrm{Pb}$ collisions after the baseline subtraction. Therefore, no modification of the azimuthal correlation of $\mathrm{D}$ mesons and charged particles in $\mathrm{p}-\mathrm{Pb}$ collisions is observed with the current uncertainties.

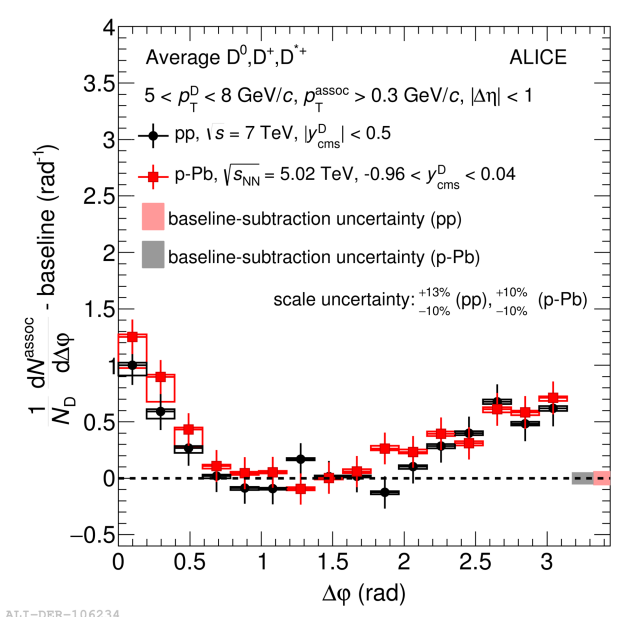

Figure 9: Comparison of the azimuthal angular correlation, after the baseline subtraction, of D mesons in the interval $5<p_{\mathrm{T}}<8 \mathrm{GeV} / c$ and charged particles for $p_{\mathrm{T}}>0.3 \mathrm{GeV} / c$ in $\mathrm{p}-\mathrm{Pb}$ collisions at $\sqrt{s_{\mathrm{NN}}}=5.02$ $\mathrm{TeV}$ and in pp collisions at $\sqrt{s}=7 \mathrm{TeV}$ [37]. 


\section{Conclusions}

We presented the ALICE results on the nuclear modification factor, elliptic flow, and azimuthal angular correlations of D mesons and electrons from heavy-flavour decays at mid-rapidity and muons from heavy-flavour decays at forward rapidity.

No significant modification of the spectra of D mesons and of electrons and muons from heavy-flavour hadron decays is observed in $\mathrm{p}-\mathrm{Pb}$ collisions relative to $\mathrm{pp}$ collisions, while results in central and semi-central $\mathrm{Pb}-\mathrm{Pb}$ collisions reveal a strong suppression for $p_{\mathrm{T}}>3 \mathrm{GeV} / c$. Therefore, no significant CNM effect on the heavy-flavour particle production is found and the suppression observed in $\mathrm{Pb}-\mathrm{Pb}$ collisions is predominantly due to the strong final-state effects of the created medium. The comparison of the $R_{\mathrm{AA}}$ of $\mathrm{D}$ mesons and non-prompt $\mathrm{J} / \psi$ from $\mathrm{B}$-hadron decays indicates a difference in the suppression of particles originating from charm and beauty quarks in the most central collisions. The measurement of the $R_{\mathrm{AA}}$ of electrons from beauty-hadron decays in the $20 \%$ most central $\mathrm{Pb}-\mathrm{Pb}$ collisions shows a suppression for $p_{\mathrm{T}}>3 \mathrm{GeV} / c$, which provides a hint for the in-medium energy loss of beauty quarks.

A positive $v_{2}$ of prompt $\mathrm{D}^{0}$ mesons is observed with more than $5 \sigma$ significance in the interval $2<p_{\mathrm{T}}<6 \mathrm{GeV} / c$ in the $30-50 \%$ centrality class, which indicates a collective motion of low- $p_{\mathrm{T}}$ charm quarks in the medium. The measured $v_{2}$ of electrons and muons from heavy-flavour hadron decays is positive with more than $3 \sigma$ significance in the intervals $1<p_{\mathrm{T}}^{\mathrm{e} \leftarrow H F}<3 \mathrm{GeV} / c$ and 3 $<p_{\mathrm{T}}^{\mu \leftarrow H F}<5.5 \mathrm{GeV} / c$ in $\mathrm{Pb}-\mathrm{Pb}$ collisions at $\sqrt{s_{\mathrm{NN}}}=2.76 \mathrm{TeV}$ in the $20-40 \%$ centrality class, indicating that heavy quarks participate in the collective motion of the medium. The centrality dependence of $v_{2}$ of the measured heavy-flavour particles hints for an increase from central to peripheral collisions, which is consistent with the qualitative expectation of the evolution of the geometrical anisotropy of the overlap region of the colliding nuclei with the centrality.

The azimuthal angular correlations between electrons from heavy-flavour hadron decays and charged particles provide the relative contribution of electrons from beauty-hadron decays to the yield of electrons from heavy-flavour decays. The obtained relative contribution in pp collisions at $\sqrt{s}=2.76 \mathrm{TeV}$ increases with $p_{\mathrm{T}}$ and reaches $50 \%$ at approximately $4 \mathrm{GeV} / c$. No modification of the azimuthal angular correlation between $\mathrm{D}$ mesons and charged particles in $\mathrm{p}-\mathrm{Pb}$ collisions at $\sqrt{s_{\mathrm{NN}}}=5.02 \mathrm{TeV}$ is observed, with the current uncertainties, with respect to the measured one in pp collisions at $\sqrt{s}=7 \mathrm{TeV}$.

\section{References}

[1] F. Karsch, J. Phys. Conf. Ser. 46 (2006) 122-131.

[2] P. Petreczky, arXiv:1301.6188 [hep-lat] (2013).

[3] Yu. L. Dokshitzer and D. E. Kharzeev, Phys. Lett. B 519 (2001) 199-206.

[4] N. Armesto, C. Salgado, and U. Wiedemann, Phys. Rev. D 69 (2004) 114003.

[5] S. Wicks, W. Horowitz, M. Djordjevic, and M. Gyulassy, Nucl. Phys. A 783 (2007) 493-496.

[6] K. Eskola, H. Paukkunen, and C. Salgado, J. High Energy Phys. 04 (2009) 065.

[7] D. de Florian and R. Sassot, Phys. Rev. D 69 (2004) 074028.

[8] H. Fujii and K. Watanabe, Nucl. Phys. A 915 (2013) 1. 
[9] CMS Collaboration, Phys. Lett. B 718 (2013) 795-814.

[10] ALICE Collaboration, J. Inst. 3 (2008) S08002.

[11] ALICE Collaboration, Int. J. Mod. Phys. A 29 (2014) 1430044.

[12] ALICE Collaboration, J. High Energy Phys. 03 (2016) 81.

[13] M. Cacciari, M. Greco, and P. Nason, J. High Energy Phys. 9805 (1998) 7 ; M. Cacciari, S. Frixione and P. Nason, J. High Energy Phys. 0103 (2001) 6.

[14] ALICE Collaboration, Phys. Rev. D 86 (2012) 112007.

[15] ALICE Collaboration, Phys. Lett. B 753 (2016) 41-56.

[16] CMS Collaboration, CMS-PAS-HIN-12-017.

[17] M. L. Miller, K. Reygers, S. J. Sanders, and P. Steinberg, Ann. Rev. Nucl. Part. Sci. 57, 205 (2007).

[18] R. Averbeck, N. Bastid, Z. Conesa del Valle, P. Crochet, and A. Dainese, X. Zhang, arXiv:1107.3243 [hep-ph] (2011).

[19] ALICE Collaboration, Phys. Lett. B 754 (2016) 81-93.

[20] ALICE Collaboration, Phys. Rev. Lett. 109 (2012) 112301.

[21] ALICE Collaboration, Phys. Lett. B 736 (2014) 196-207.

[22] CMS Collaboration, J. High Energy Phys. 1205 (2012) 063.

[23] M. Djordjevic, M. Djordjevic, and B. Blagojevic, Ó Phys. Lett. B 737 (2014) $298 Đ 302$.

[24] A. Andronic, F. Arleo, R. Arnaldi, A. Beraudo, E. Bruna, et al., arXiv:1506.03981 [nucl-ex] (2015).

[25] Z.-B. Kang, I. Vitev, E. Wang, H. Xing, and C. Zhang, Phys. Lett. B 740 (2015) 23-29.

[26] R. Sharma, I. Vitev, and B.-W. Zhang, Phys. Rev. C 80 (2009) 054902.

[27] A.M. Sickles, Phys. Lett. B 731 (2014) $51 Đ 56$.

[28] K.J. Eskola, H. Paukkunen, and C.A. Salgado, J. High Energy Phys. 04 (2009) 065.

[29] ALICE Collaboration, Phys. Rev. Lett. 111 (2013) 102301.

[30] ALICE Collaboration, Phys. Rev. C 90 (2014) 034904.

[31] ALICE Collaboration, arXiv:1606.00321 [nucl-ex] (2016).

[32] J. Uphoff, O. Fochler, Z. Xu, and C. Greiner, Phys. Lett. B 717 (2012) 430-435.

[33] M. He, R. J. Fries, and R. Rapp, arXiv:1208.0256 [nucl-th] (2012).

[34] M. Monteno, W. M. Alberico, A. Beraudo, A. De Pace, A. Molinari, M. Nardi, and F. Prino, J. Phys. G. 38 (2011) 124144.

[35] P. B. Gossiaux, R. Bierkandt, and J. Aichelin, Phys. Rev. C 79 (2009) 044906.

[36] ALICE Collaboration, arXiv:1405.4144 [nucl-ex] (2014).

[37] ALICE Collaboration, arXiv:1605.06963 [nucl-ex] (2016).

[38] T. Sjostrand, S. Mrenna, and P. Z. Skands, J. High Energy Phys. 05 (2006) 26.

[39] P. Bolzoni and G. Kramer, Nucl. Phys. B 872 (2013) 253.

[40] R. Maciula and A. Szczurek, Phys. Rev. D 879 (2013) 094022.

[41] ALICE Collaboration, arXiv:1405.4117 [nucl-ex] (2014). 